Relations industrielles

Industrial Relations

\title{
Bernier, Robert, et James Iain Gow, dir., Un État réduit? A Down-Sized State?
}

\section{Gérard Ouimet}

Volume 51, numéro 1, 1996

URI : https://id.erudit.org/iderudit/051089ar

DOI : https://doi.org/10.7202/051089ar

Aller au sommaire du numéro

Éditeur(s)

Département des relations industrielles de l'Université Laval

ISSN

0034-379X (imprimé)

1703-8138 (numérique)

Découvrir la revue

Citer ce compte rendu

Ouimet, G. (1996). Compte rendu de [Bernier, Robert, et James Iain Gow, dir., Un État réduit? A Down-Sized State?]. Relations industrielles / Industrial

Relations, 51(1), 224-225. https://doi.org/10.7202/051089ar

Tous droits réservés (C) Département des relations industrielles de l'Université Laval, 1996
Ce document est protégé par la loi sur le droit d'auteur. L'utilisation des services d'Érudit (y compris la reproduction) est assujettie à sa politique d'utilisation que vous pouvez consulter en ligne.

https://apropos.erudit.org/fr/usagers/politique-dutilisation/ 


\section{Un Etą réduit ?/A Down-Sized State?}

sous la direction de Robert BERNIER et de James Iain Gow, Sainte-Foy, Presses de l'Université du Québec, 1994, 435 p., ISBN 2-7605-0740-8.

D'entrée de jeu, il convient de reconnaître que l'ouvrage produit sous la direction de Robert Bernier et de James lain Gow constitue une substantielle réflexion tant sur la nature que sur le devenir de l'appareil étatique canadien et québécois. Les thématiques afférentes à la problématique de l'atrophie ou du désengagement de l'État sont à la fois diversifiées et fort congrues eu égard à la contemporanéité du phénomène politique observé. Ces thématiques, au nombre de huit, sont : la gestion financière, la fonction publique, la déréglementation, la privatisation des entreprises publiques, l'évaluation des programmes, l'information et le marketing gouvernemental, la gestion centrale et le rôle de l'État.

La totalité des études regroupées dans les diverses thématiques examine l'hypothèse de la réduction, ou du moins de la stagnation, de la taille de l'État et de ses nombreux appendices. En dépit de la diversité des sujets d'études abordés par les auteurs, une tendance lourde dans la démarche analytique empruntée par ceux-ci ressort clairement, à savoir l'utilisation d'une approche descriptive, faisant appel à des indicateurs d'ordre quantitatif. C'est ainsi que les études entre autres de Prince (Canada's Public Finances under Restraint : Has Ottawa Shrunk?), Beauregard (Les finances publiques du Québec et le désengagement de l'État au cours des années 80), Simard (L'État réduit: le cas de la fonction publique fédérale), Gow (La gestion des ressources humaines dans une période de compressions budgétaires : la fonction publique du Québec, de 1981 à 1991), Bourgault et Dion (La haute fonction publique a-t-elle essuyé sa part des compressions ?) Stanbury (Privatization by Federal and Provincial Governments in Canada : An Empirical Study), Marceau (L'évaluation de programmes : rationalisation des dépenses ou stratégie de marketing) et Mercier et Bourgeois (Dix ans de restrictions budgétaires au gouvernement du Québec : organismes centraux et tendances administratrives, 1981, 1991 ) brossent, tant aux niveaux ponctuel que longitudinal, un tableau des vicissitudes de l'État abondamment documenté par des indices statistiques dont la signification et la portée ne sont plus à démontrer. Le travail réalisé par ces auteurs est d'une rigueur démonstrative peu commune. D'ailleurs, bon nombre de ceux-ci construisent leur argumentation sous un mode analytique cartésien. À titre exemplatif, il convient ici de reporter le tout début du texte de Bourgault et Dion (chapitre 5, p. 103-104), à savoir :

La haute fonction publique essuie-telle sa part des compressions? À cette question, on peut donner trois réponses simples. On peut affirmer que les coupes dans les effectifs et les rémunérations :

1. frappent moins...

2. frappent davantage...

3. frappent autant..

... les grands que les moyens et les petits fonctionnaires.

Laquelle de ces trois affirmations traduit le mieux la réalité ?

Pareil procédé facilite de beaucoup la clarté de la démonstration : le champ d'investigation étant, on ne peut plus, balisé. Ce découpage argumentaire constitue assurément la plus grande qualité de l'ouvrage. Dès les premiers paragraphes de chacun des chapitres, le lecteur connaît précisément la problématique soumise à l'étude ainsi que la démarche analytique empruntée pour l'ausculter. C'est un des rares ouvrages, sondant les composantes tentaculaires d'un objet d'analyse fort complexe, l'État et sa périphérie, qui permet une compréhension à la fois accessible, significative et étayée de la mouvance de l'État. C'est ainsi que 
nonobstant la rigueur et la solidité des assises factuelles à la base des démonstrations entreprises par les différents auteurs du collectif, l'ouvrage s'adresse à un vaste public et risque d'en intéresser plus d'un.

Par ailleurs, l'ouvrage possède les défauts de ses qualités, à savoir que sa teneur analytique essentiellement descriptive empêche l'approfondissement des réflexions amorcées, soit un questionnement d'ordre explicatif. Quoique la recherche du comment des choses s'avère une démarche cognitive de toute première importance, elle ne permet pas de saisir la dynamique d'un phénomène, en l'occurrence le désengagement de l'État. Il n'y a pas de véritables tentatives d'explications arcboutées sur la construction d'une problématique interpellant le pourquoi des choses. Pourquoi le désengagement de l'État à une époque où les forces conjoncturelles (mondialisation de l'économie, exacerbation des nationalismes et pressions démographiques) en font un acteur hautement stratégique, voire central? Qui plus est, une telle recherche des causes de l'êtiolement du rôle et de la place de l'État est considérablement minée par l'absence d'une utilisation systématique d'un modèle théorique traitant de l'objet investigué. Pourtant, ce ne sont pas les modèles théoriques en science politique qui manquent à cet effet ! Nul n'est besoin de recourir à des conceptualisations tarabiscotées de l'État, tels, par exemple, les travaux d'Amin ou de Poulantzas. Un cadre d'analyse aussi simple que celui proposé par Bartha ("Issues Management : Theory and Practice ", M.C. Baetz (ed), Readings and Canadian Cases in Business, Government, and Society, Scarborough, Nelson Canada, 1993) aurait permis une appréhension des motivations à la base des comportements des acteurs.

De plus, l'ouvrage souffre d'un certain syncrétisme. Encore que tous les textes convergent vers une réflexion centrale, le champ d'action de l'État, il n'y a pas pour autant entre eux de fil conducteur. L'éclatement synthétique de l'ouvrage aurait pu être compensé par des entrées en matière à chacun des chapitres rédigées par les directeurs de l'ouvrage. De même, une conclusion synoptique à la toute fin de l'ouvrage aurait grandement contribué au resserrement de la synthèse.

Nonobstant ces quelques éléments lacunaires, nous sommes indubitablement en présence d'un ouvrage de grande qualité, fort bien rédigé et substantiellement documenté, qui jette une lumière éclairante et originale sur la nature de l'appareil étatique. À l'instar des travaux de McRoberts ou de Coleman, cet ouvrage s'avère une référence incontournable pour quiconque s'interroge sérieusement sur l'évolution du rôle de l'État canadien ou québécois.

\section{GÉRARD OUIMET École des Hautes Études Commerciales}

